\title{
Hysterectomy with salpingectomy versus hysterectomy alone
}

DOI:

10.1002/14651858.CD012858

\section{Document Version}

Final published version

Link to publication record in Manchester Research Explorer

\section{Citation for published version (APA):}

van Lieshout, L. A. M., Steenbeek, M. P., De Hullu, J. A., Vos, M. C., Houterman, S., Wilkinson, J., \& Piek, J. M. J. (2017). Hysterectomy with salpingectomy versus hysterectomy alone. Cochrane Database of Systematic Reviews, 2017(11), [CD012858]. https://doi.org/10.1002/14651858.CD012858

\section{Published in:}

Cochrane Database of Systematic Reviews

\section{Citing this paper}

Please note that where the full-text provided on Manchester Research Explorer is the Author Accepted Manuscript or Proof version this may differ from the final Published version. If citing, it is advised that you check and use the publisher's definitive version.

\section{General rights}

Copyright and moral rights for the publications made accessible in the Research Explorer are retained by the authors and/or other copyright owners and it is a condition of accessing publications that users recognise and abide by the legal requirements associated with these rights.

\section{Takedown policy}

If you believe that this document breaches copyright please refer to the University of Manchester's Takedown Procedures [http://man.ac.uk/04Y6Bo] or contact uml.scholarlycommunications@manchester.ac.uk providing relevant details, so we can investigate your claim.

\section{OPEN ACCESS}




\section{(7) Cochrane Library}

Cochrane Database of Systematic Reviews

\section{Hysterectomy with salpingectomy versus hysterectomy alone (Protocol)}

van Lieshout LAM, Steenbeek MP, De Hullu JA, Vos MC, Houterman S, Wilkinson J, Piek JMJ

www.cochranelibrary.com 


\section{TABLE OF CONTENTS}

HEADER . . . . . . . . . . . . . . . . . . . . . . . . . . . . . . . . . . . . . . . . 1

ABSTRACT . . . . . . . . . . . . . . . . . . . . . . . . . . . . . . . . . . . . . . . 1

BACKGROUND . . . . . . . . . . . . . . . . . . . . . . . . . . . . . . . . . . . . . . . 1

OBJECTIVES . . . . . . . . . . . . . . . . . . . . . . . . . . . . . . . . . . . . . . 2

METHODS . . . . . . . . . . . . . . . . . . . . . . . . . . . . . . . . . . . . . . 2

ACKNOWLEDGEMENTS . . . . . . . . . . . . . . . . . . . . . . . . . . . . . . . . . . . . . . .

REFERENCES . . . . . . . . . . . . . . . . . . . . . . . . . . . . . . . . . . . . . . . . . 6

APPENDICES . . . . . . . . . . . . . . . . . . . . . . . . . . . . . . . . . . . . . . . . . . . . . . . .

CONTRIBUTIONS OF AUTHORS . . . . . . . . . . . . . . . . . . . . . . . . . . . . . . . . . . . . . . . . . . . 12

DECLARATIONS OF INTEREST . . . . . . . . . . . . . . . . . . . . . . . . . . . . . . . 12

SOURCES OF SUPPORT . . . . . . . . . . . . . . . . . . . . . . . . . . . . . . . . . . 12 
[Intervention Protocol]

\section{Hysterectomy with salpingectomy versus hysterectomy alone}

Laura A M van Lieshout ${ }^{1 a}$, Miranda P Steenbeek ${ }^{2 b}$, Joanne A De Hullu ${ }^{2}$, M Caroline Vos ${ }^{3}$, Saskia Houterman ${ }^{4}$, Jack Wilkinson ${ }^{5}$, Jurgen MJ Piek ${ }^{1}$

${ }^{1}$ Department of Obstetrics and Gynaecology, Catharina Cancer Institute, Catharina Hospital, Eindhoven, Netherlands. ${ }^{2}$ Department of Obstetrics and Gynaecology, Radboud University Nijmegen Medical Centre, Nijmegen, Netherlands. ${ }^{3}$ Obstetrics and Gynaecology, Elisabeth-TweeSteden Hospital, Tilburg, Netherlands. ${ }^{4}$ Department of Education and Research, Catharina Hospital, Eindhoven, Netherlands. ${ }^{5}$ Centre for Biostatistics, School of Health Sciences, Faculty of Biology, Medicine and Health, Manchester Academic Health Science Centre (MAHSC), University of Manchester, Manchester, UK

${ }^{a}$ Dual first author. ${ }^{b}$ Dual first author

Contact address: Jurgen MJ Piek, Department of Obstetrics and Gynaecology, Catharina Cancer Institute, Catharina Hospital, Michelangelolaan 2, Eindhoven, 5623EJ, Netherlands. jurgen.piek@catharinaziekenhuis.nl.

Editorial group: Cochrane Gynaecology and Fertility Group.

Publication status and date: New, published in Issue 11, 2017.

Citation: van Lieshout LAM, Steenbeek MP, De Hullu JA, Vos MC, Houterman S, Wilkinson J, Piek JMJ. Hysterectomy with salpingectomy versus hysterectomy alone. Cochrane Database of Systematic Reviews 2017, Issue 11. Art. No.: CD012858. DOI: 10.1002/14651858.CD012858.

Copyright (C) 2017 The Cochrane Collaboration. Published by John Wiley \& Sons, Ltd.

\section{A B S T R A C T}

This is a protocol for a Cochrane Review (Intervention). The objectives are as follows:

To evaluate the effect of bilateral salpingectomy during hysterectomy on:

1. the incidence of epithelial ovarian cancer;

2. surgery-related adverse events;

3. postoperative ovarian function and ovarian reserve.

\section{B A C K G R O U N D}

\section{Description of the condition}

Ovarian cancer is the gynaecological malignancy with the highest mortality rate; it has an overall five-year survival rate of 30 to 40\%.(Bolton 2012; Siegel 2017) This dismal prognosis is mainly the result of non-specific symptoms, leading to detection at an advanced stage of disease. Despite progress over the past decades in the field of cancer treatment in general, only limited improvements have been made in ovarian cancer. Studies aimed at the detection of ovarian cancer at an early stage of disease failed to show survival benefit. Hence, preventive measures that are both safe and effective are needed. Currently, the only option for the prevention of ovarian cancer is bilateral salpingo-oophorectomy (BSO; the removal of both of the ovaries and the fallopian tubes), which reduces the risk of ovarian cancer by $80 \%$ to $96 \%$ (Kauff 2008; Rebbeck 2009). However, BSO results in immediate menopause, which in turn 
leads to elevated risks of, for example, ischaemic heart disease and an all-cause mortality (Mytton 2017; Parker 2009; Rocca 2006).

\section{Description of the intervention}

A bilateral salpingectomy is defined as the surgical excision of both fallopian tubes, up to the tubal corner of the uterus. The procedure can be implemented in several ways, for example during a hysterectomy (the removal of the uterus), a common treatment for both benign and malignant gynaecological conditions. The surgical approach taken during hysterectomy can be vaginal, per laparotomy or per laparoscopy. Possible additional complications of the salpingectomy procedure include an increased chance of excessive blood loss, infection or damage to adjacent visceral organs.

\section{How the intervention might work}

Over the past two decades, it has become apparent and more commonly accepted that epithelial ovarian cancer, the most common histological subtype of ovarian cancer, probably arises from the epithelium of the fallopian tube rather than from the ovary itself (Chen 2017; Kindelberger 2007; Perez-Lopez 2017; Piek 2001a; Piek 2003). This insight has given rise to the hypothesis that salpingectomy, after the completion of childbearing, may reduce the risk of epithelial ovarian cancer (Chen 2017; Kindelberger 2007; Long 2017; Perez-Lopez 2017). One suggestion has been to combine salpingectomy with hysterectomy for benign gynaecological conditions, but there is concern that this could lead to an increase in surgical complications. Additionally, salpingectomy could affect the ovarian reserve since the ovaries and the fallopian tubes (partially) share a blood supply. Thus, excision of the fallopian tubes could harm the ovarian blood supply and affect ovarian function and the ovarian reserve.

\section{Why it is important to do this review}

Since 2001 accumulating evidence points towards the epithelium of the fallopian tubes as a precursor site for epithelial ovarian cancer (Chen 2017; Kindelberger 2007; Long 2017; Perez-Lopez 2017; Piek 2001a; Piek 2001b). In some countries, this insight has already resulted in the implementation of opportunistic salpingectomies in women undergoing hysterectomy for benign gynaecological conditions. The Royal College of Obstetricians and Gynaecologists, the American College of Obstetricians and Gynecologists, and the European Menopause and Andropause Society each recently published statements on the importance of discussing the possibility of opportunistic salpingectomy with women undergoing hysterectomy for benign gynaecological conditions. However, they also stated that more research on the topic is needed, since it remains to be elucidated whether opportunistic salpingectomy will really result in a decreased incidence of epithelial ovarian cancer and whether opportunistic salpingectomy is safe (primum non nocere) (ACOG 2015; Perez-Lopez 2017; RCOG 2014).

The aim of this review will be to summarise and analyse the current literature on both the expected health gain and risks of carrying out salpingectomy during hysterectomy for benign gynaecological conditions.

\section{O B J E C T I V E S}

To evaluate the effect of bilateral salpingectomy during hysterectomy on:

1. the incidence of epithelial ovarian cancer;

2. surgery-related adverse events;

3. postoperative ovarian function and ovarian reserve.

\section{METHODS}

\section{Criteria for considering studies for this review}

\section{Types of studies}

Because of the relatively low incidence of the ovarian cancer and the necessity of a follow-up spanning several decades, our first listed objective, the effect of salpingectomy on the incidence of epithelial ovarian cancer, is not a particularly suitable outcome for a randomised controlled trial (RCT). For this objective, we will therefore consider both RCTs and non-randomised trials (NRTs) included in this review. Since the risk of bias is greater in NRTs than in RCTs, we will consider only cohort studies (both retrospective and prospective), case-control studies and cross-sectional studies. Although the existence of RCTs and quasi-randomised trials is highly unlikely, we will assess them for inclusion if available.

Because our second and third objectives, the effect of salpingectomy on the incidence of surgery-related adverse events, and on postoperative ovarian function and ovarian reserve, are suitable outcomes for RCTs, for these objectives we will consider only RCTs included in this review.

\section{Types of participants}

Individuals with a population-based risk of ovarian cancer undergoing surgery for benign gynaecological conditions. We will exclude trials that include the following types of women:

- women with a history of ovarian cancer;

- women with an elevated risk of ovarian cancer based on a proven gene mutation; 
- women who have undergone previous bilateral oophorectomy;

- women who have undergone previous bilateral salpingectomy.

The exclusion of women with a proven germline BRCA $1 / 2$ gene mutation is important since there are limited data available to suggest that mutation carriers may undergo an earlier menopause than the general population (Finch 2013).

\section{Types of interventions}

We will include both RCTs and NRTs that compare hysterectomy with bilateral salpingectomy to hysterectomy with preservation of the fallopian tubes.

\section{Types of outcome measures}

\section{Primary outcomes}

For RCTs and NRTs

1. Incidence of epithelial ovarian cancer.

- Epithelial ovarian cancer is defined as a pathologically confirmed malignancy derived from the ovary or fallopian tube.

\section{For RCTs}

2. Surgery-related adverse events.

- Intraoperative complications (including injuries to the bladder, ureters, intestines, blood vessels, nerves and excessive blood loss).

- Short-term postoperative complications (including vascular, wound, gastrointestinal, neurological, respiratory and urinary tract complications).

\section{Postoperative hormonal status.}

- Preferably by assessment of postoperative anti-müllerian hormone (AMH) concentrations (van Rooij 2005; Depmann 2016).

\section{Secondary outcomes}

For RCTs

4. Total surgical time.

5. Estimated blood loss.

6. Conversion rate to open surgery (applicable only to laparoscopic and vaginal approaches).

7. Duration of hospital admission.

8. Menopause-related symptoms.

9. Quality of life.

\section{Search methods for identification of studies}

We will search for all published and unpublished studies investigating bilateral salpingectomy during hysterectomy, without language restriction. in consultation with On Ying Chan (Radboud University Information Specialist) and Marian Showell (Cochrane Gynaecology and Fertility Group (CGF) Information Specialist).

\section{Electronic searches}

We will search for papers published in all languages and, if necessary, will obtain translations. We will search the following databases, from their inception until present, using a combined strategy that has been developed:

- Cochrane Gynaecology and Fertility Group (CGF) Specialised Register of Controlled Trials, PROCITE platform (Appendix 1)

- Cochrane Central Register of Controlled Trials

(CENTRAL) Cochrane Library platform (Appendix 2);

- MEDLINE (Epub Ahead of Print, In-Process \& other Non-indexed Citations) Ovid platform (Appendix 3);

- Embase Ovid platform (Appendix 4);

- PsycINFO Ovid platform (Appendix 5);

- CINAHL Ebsco platform (Appendix 6).

Additionally, we will search for ongoing and registered trials ( Appendix 7) in the following ongoing trial registries:

- clinicaltrials.gov/ (a service of the US National Institutes of Health);

- who.int/trialsearch/default.aspx (the World Health Organization International Trials Registry Platform search portal).

\section{Searching other resources}

We will handsearch the reports of conferences from the following sources: ESGO (European Society of Gynaecological oncology), SGO (Society of Gynecological Oncology), ESHRE (European Society of Human Reproduction and Embryology), EMAS (European Menopause and Andropause Society) and IMS (International Menopause Society). To identify additional trials, we will handsearch the reference lists of all relevant trials obtained by the initial search. We will limit this search to articles and reports published since 1997, as the fallopian tube has been considered as the origin of epithelial ovarian cancer only since 2001 Piek 2001a.

\section{Data collection and analysis}

\section{Selection of studies}

We will import the titles and abstracts retrieved by the search into the reference manager database Covidence (Covidence). Two 
review authors (LL, MS) will independently screen the references and check them for duplicates. After obtaining the full text versions of potentially relevant studies, two review authors (LL, MS) will independently assess their eligibility for inclusion. We will resolve any disagreements by discussion and, if necessary, by consulting a third review author (JP). Where the judgement of a review author could be biased due to a conflict of interest, one of the other review authors will assess that particular study. We will document reasons for exclusion and the selection process in a PRISMA flow chart.

\section{Data extraction and management}

We will predesign a data extraction form for the extraction of relevant data from included trials. Prior to data extraction, two review authors (LL, MS) will perform an independent trial run of the data extraction form on a sample of studies. Two review authors (LL, MS) will independently extract data on the number of participants, characteristics of participants, characteristics of the intervention with and without salpingectomy, study quality, duration of follow-up and outcomes. We will resolve any disagreements by discussion and, if necessary, by consulting a third review author (JP). We will attempt to retrieve missing data by contacting the study authors. For studies with multiple publications, we will collate multiple reports of the same study so that each study, rather than each report, is the unit of interest in the review. We will assign these studies a single study identifier.

\section{Assessment of risk of bias in included studies}

Two review authors (JW, MS) will independently assess the methodological quality of included studies; we will resolve disagreements by consulting a third review author (JP).

For non-randomised studies, we will assess the likelihood of bias according to the ROBINS-I (a tool for assessing the risk of bias in non-randomised studies of interventions) (Sterne 2016). The hypothetical 'target' trial necessary for the use of the ROBINSI would be a large RCT in which women would be allocated to the intervention group (i.e. hysterectomy with bilateral salpingectomy) or the control group (i.e. hysterectomy without bilateral salpingectomy). Baseline information on both groups should include age, parity, the use of oral contraceptives and surgical history. During a follow-up period of at least 40 years, family history (breast and ovarian cancer), age at menopause, use of oral contraceptives, abdominal surgery and the occurrence of epithelial ovarian cancer should have been documented. If a participant is diagnosed with epithelial ovarian cancer, data on age at diagnosis, tumour stage and histology of the primary tumour should have been collected.

We will assess the eligible studies for bias due to confounding, selection of participants, classification of interventions, missing data, measurement of outcomes and selection of the reported result. We have identified the following domains as potential confounders, which therefore should preferably be similar among study groups: age, parity, family history of ovarian or breast cancer, use of oral contraception and history of tubal ligation. We have identified no co-interventions that could potentially confound the results. Confounding might result in considerable heterogeneity between studies and requires adequate methods to control for it, such as stratification of regression modelling with propensity scores or covariates. We will assess the appropriateness and quality of these methods critically. We will compare NRTs to their published protocol, where available, so as to assess selective or incomplete reporting.

We will assess the risk of bias in randomised studies using the Cochrane's 'Risk of bias' assessment tool (Higgins 2011) and will include the following domains: random sequence generation, allocation concealment, blinding of participants and personnel, blinding of outcome assessment, incomplete outcome data and selective reporting. If available, we will compare the published protocols of selected studies to the reported outcomes so as to assess selective or incomplete reporting bias.

\section{Measures of treatment effect}

For NRTs, we will extract and report both unadjusted and adjusted effect estimates. For cohort studies, we will calculate a hazard ratio (HR). We expect there to be a long duration of follow-up for the epithelial ovarian cancer outcome, which might result in selection bias over time. We will, therefore, calculate HRs for different timepoints. For case-control studies, we will calculate odds ratios (OR) with $95 \%$ confidence intervals (CIs) by extracting the number of participants in each treatment arm that experienced the outcome of interest, and the number of participants assessed per outcome. For dichotomous data extracted from RCTs (i.e. adverse surgical events), we will calculate ORs with 95\% CIs. For continuous data (i.e. postoperative hormonal status), we will estimate mean differences (MDs) with 95\% CIs for variables with a normal distribution where the same measure was used to assess the outcome. If the included studies used different measures to assess the same outcome, we will use the standardised mean difference (SMD). For skewed continuous variables, we will extract mean values and standard deviations. If necessary, we will transform the data prior to meta-analysis according to one of three methods presented by Higgins 2008a.

If the data necessary to calculate ORs or MDs are not available, we will make use of the most detailed numerical data available that may facilitate similar analyses of included studies. We will attempt to retrieve missing data by contacting the study authors.

\section{Unit of analysis issues}

We will perform the primary analysis per woman included in the studies. 


\section{Dealing with missing data}

For NRTs, we will conduct sensitivity analyses to assess how robust our conclusions are to assumptions about missing data (Higgins 2008b).

For RCTs, we will analyse the retrieved data according to the intention-to-treat principle. In case of missing data, we will contact the original researchers to obtain the missing data. If these attempts do not provide us with extra data, we will use only the data that are available. We will perform an additional per-protocol analysis, if applicable.

\section{Assessment of heterogeneity}

For NRTs, we expect heterogeneity, and thus we will base our assessment of heterogeneity on consideration of the different study designs and analysis details.

To examine whether meta-analysis is possible for RCTs, we will assess the heterogeneity of the included studies using the $\mathrm{I}^{2}$ statistic. This heterogeneity is important, since it indicates whether the studies are similar in clinical and methodological characteristics. We will consider an $\mathrm{I}^{2}$ value of $50 \%$ or higher as indicating substantial heterogeneity.

\section{Assessment of reporting biases}

In view of the difficulty of detecting and correcting for publication bias and other reporting biases, we will aim to minimise their potential impact by performing an extensive search for eligible studies and by being alert for the duplication of data. If there are 10 or more studies in an analysis, we will use a funnel plot to explore the possibility of small study effects (the tendency for estimates of the intervention effect to be more beneficial in smaller studies). To prevent language bias, we will exclude no studies based on language. If the studies prove to be exceptionally difficult to translate, we will ask the authors to provide a summary of their methods and results. We will compare the studies, and authors and their affiliations so as to avoid multiple publication bias.

\section{Data synthesis}

If the selected studies are considered to be similar enough for metaanalysis, we will combine the data using a fixed-effect model. We will make the following comparisons.

1. Incidence of epithelial ovarian cancer after hysterectomy with bilateral salpingectomy versus incidence of epithelial ovarian cancer after hysterectomy without bilateral salpingectomy.

2. Surgical outcomes of hysterectomy with bilateral salpingectomy versus hysterectomy without bilateral salpingectomy.

3. Ovarian reserve after hysterectomy with bilateral salpingectomy versus hysterectomy without bilateral salpingectomy.

\section{Subgroup analysis and investigation of heterogeneity}

Where possible, depending on the availability of the data, we will perform the following subgroup analyses.

1. Effect of salpingectomy on the incidence of epithelial ovarian cancer in the following subgroups:

a. premenopausal versus postmenopausal women.

2. Effect of salpingectomy on the incidence of epithelial ovarian cancer in the following subgroups:

a. nulliparous versus parous women.

3. Incidence of epithelial ovarian cancer in women who have a history of tubal ligation versus incidence of epithelial ovarian cancer in women who have no history of tubal ligation.

4. Incidence of surgery-related adverse events depending on surgical approach:

a. abdominal approach versus laparoscopic approach;

b. vaginal approach versus laparoscopic approach;

c. abdominal approach versus vaginal approach.

\section{Sensitivity analysis}

For the primary outcomes, we will conduct sensitivity analyses to determine whether the review conclusion would remain the same if:

- eligibility had been restricted to studies without high risk of bias (which we define as those with no high risk of bias in any domain);

- a random-effects model had been adopted;

- NRTs had been excluded (only applicable if RCTs have been included).

\section{Overall quality of the body of evidence: 'Summary of findings' table}

We will prepare a 'Summary of findings' table using GRADEpro and Cochrane methods (GRADEpro GDT 2015). This table will evaluate the overall quality of the body of evidence for the main review outcomes (incidence of epithelial ovarian carcinoma, surgeryrelated adverse events and postoperative hormonal status) for the main review comparison (hysterectomy with salpingectomy versus hysterectomy without salpingectomy). If appropriate, we will prepare additional 'Summary of findings' tables for the main review outcomes of other important comparisons (premenopausal versus postmenopausal women, nulliparous women versus parous women, women with a history of tubal ligation versus women with no history of tubal ligation, laparoscopic approach versus abdominal approach). We will assess the quality of the evidence using GRADE criteria: risk of bias, consistency of effect, imprecision, indirectness and publication bias. Two review authors (LL, MS) working independently will make judgements about the evidence quality (high, moderate, low or very low); we will resolve disagreements by discussion. We will justify, document and incorporate all judgements into the report of results for each outcome. 
We plan to extract study data, format our comparisons in data tables and prepare a 'Summary of findings' table before writing the results and conclusions of our review.

\section{ACK NOWLEDGEMENTS}

Marian Showell (Cochrane Gynaecology and Fertility Group (CGF) Information Specialist), On Ying Chan (Radboud University Information Specialist) and Godelieve Engbersen (ElisabethTweesteden Hospital Information SpecialisT) for conducting the electronic search.

\section{REFERE N C ES}

\section{Additional references}

\section{ACOG 2015}

Committee on Gynecologic Practice. Committee opinion no. 620: Salpingectomy for ovarian cancer prevention. Obstetrics \& Gynecology 2015;125(1):279-81.

Bolton 2012

Bolton KL, Chenevix-Trench G, Goh C, Sadetzki S, Ramus SJ, Karlan BY, et al. Assosciation Between BRCA1 and BRCA2 Mutations and Survival in Women With Invasive Epithelial Ovarian Cancer. JAMA January 25, 2012;307 (4):382-390.

Chen 2017

Chen F, Gaitskell K, Garcia MJ, Albukhari A, Tsaltas J, Ahmed AA. Serous tubal intraepithelial carcinomas associated with high-grade serous ovarian carcinomas: a systematic review. BJOG 2017;124(6):872-8. [PUBMED: 28218502]

Covidence [Computer program]

Veritas Health Innovation. Covidence. Version accessed xxx. Melbourne: Veritas Health Innovation.

\section{Depmann 2016}

Depmann J, Eijkemans MJC, Broer SL, Scheffer GJ, van Rooij IA, Laven JS, et al. Does anti-Müllerian hormone predict menopause in the general population? Results of a prospective ongoing cohort study. Human Reproduction 2016;31(7):1579-87.

Finch 2013

Finch A, Valentini A, Greenblatt E, Lynch HT, Ghadirian P, Armel S, et al. Frequency of premature menopause in women who carry a BRCA1 or BRCA2 mutation. Fertily and Sterility 2013;99(6):1724-8.

GRADEpro GDT 2015 [Computer program] McMaster University (developed by Evidence Prime). GRADEpro GDT. Version accessed xxx. Hamilton (ON): McMaster University (developed by Evidence Prime), 2015.

\section{Higgins 2008a}

Higgins JP, White IR, Anzures-Cabrera J. Meta-analysis of skewed data: combining results reported on log-transformed or raw scales. Statistics in Medicine 2008;27(29):6072-92.

\section{Higgins 2008b}

Higgins JP, White IR, Wood AM. Imputation methods for missing outcome data in meta-analysis of clinical trials. Clinical Trials 2008;5(3):225-39.

\section{Higgins 2011}

Higgins JPT, Green S, editor(s). Cochrane Handbook for Systematic Reviews of Interventions Version 5.1.0 (updated March 2011). The Cochrane Collaboration, 2011. Available from handbook.cochrane.org.

\section{Kauff 2008}

Kauff ND, Domchek SM, Friebel TM, Robson ME, Lee J, Garber JE, et al. Risk-reducing salpingo-oophorectomy for the prevention of BRCA1- and BRCA2-associated breast and gynecologic cancer: a multicenter, prospective study. Journal of Clinical Oncology 2008;26(8):1331-7. [PUBMED: 18268356]

\section{Kindelberger 2007}

Kindelberger DW, Lee Y, Miron A, Hirsch MS, Feltmate $\mathrm{C}$, Medeiros F, et al. Intraepithelial carcinoma of the fimbria and pelvic serous carcinoma: evidence for a causal relationship. The American Journal of Surgical Pathology 2007;31(2):161-9. [PUBMED: 17255760]

Long 2017

Long Roche KC, Abu-Rustum NR, Nourmoussavi M, Zivanovic O. Risk-reducing salpingectomy: Let us be opportunistic. Cancer 2017;123(10):1714-20. [PUBMED: 28334425]

\section{Mytton 2017}

Mytton J, Evison F, Chilton PJ, Lilford RJ. Removal of all ovarian tissue versus conserving ovarian tissue at time of hysterectomy in premenopausal patients with benign disease: study using routine data and data linkage. BMJ (Clinical research ed.) 2017;356:j372. [PUBMED: 28167486] 
Parker 2009

Parker WH, Broder MS, Chang E, Feskanich D, Farquhar $\mathrm{C}$, Liu Z, et al. Ovarian conservation at the time of hysterectomy and long-term health outcomes in the nurses' health study. Obstetrics and Gynecology 2009;113(5): 1027-37. [PUBMED: 19384117]

\section{Perez-Lopez 2017}

Perez-Lopez FR, Ceausu I, Depypere H, Kehoe S, Lambrinoudaki I, Mueck A, et al. Interventions to reduce the risk of ovarian and fallopian tube cancer: a European Menopause and Andropause Society Postition Statement. Maturitas 2017;100:86-91. [PUBMED: 28389043]

Piek 2001a

Piek JMJ, van Diest PJ, Zweemer RP, Jansen JW, PoortKeesom RJJ, Menko FH, et al. Dysplastic changes in prophylactically removed Fallopian tubes of women predisposed to developing ovarian cancer. Journal of Pathology 2001;195:451-456.

\section{Piek 2001b}

Piek JMJ, van Diest PJ, Zweemer RP, Kenemans P, Verheijen RHM. Tubal ligation and risk of ovarian cancer. The Lancet September 8, 2001;358:844.

Piek 2003

Piek JMJ, Verheijen RHM, Kenemans P, Massuger LF, Bulten $\mathrm{H}$, van Diest PJ. BRCA1/2-related ovarian cancers are of tubal origin: a hypothesis. Gynecologic Oncology 2003;90(2):491.

\section{RCOG 2014}

Royal College of Obstetricians and Gynaecologists. The distal fallopian tube as the origin of non-uterine pelvic highgrade serous carcinomas. Scientific impact paper no. 44. November 2014. www.rog.org.uk/globalassets/documents/ guidelines/scientific-impact-papers/sip44hgscs.pdf (accessed 25 September 2017).

Rebbeck 2009

Rebbeck TR, Kauff ND, Domchek SM. Meta-analysis of risk reduction estimates associated with risk-reducing salpingo-oophorectomy in BRCA1 or BRCA2 mutation carriers. Journal of the National Cancer Institute 2009;101 (2):80-7. [PUBMED: 19141781]

\section{Rocca 2006}

Rocca WA, Grossardt BR, de Andrade M, Malkasian GD, Melton LJ 3rd. Survival patterns after oophorectomy in premenopausal women: a population-based cohort study. The Lancet. Oncology 2006;7(10):821-8. [PUBMED: 17012044]

Siegel 2017

Siegel RL, Miller KD, Jemal A. Cancer statistics, 2017. CA A Cancer Journal for Clinicians January/February 2017;67 (1):7-30.

\section{Sterne 2016}

Sterne JA, Hernan MA, Reeves BC, Savovic J, Berkman ND, Viswanathan M, et al. ROBINS-I: a tool for assessing risk of bias in non-randomised studies of interventions. BMJ (Clinical research ed.) 2016;355:i4919. [PUBMED: 27733354]

van Rooij 2005 van Rooij IAJ, Broekmans FJM, Scheffer GJ, Looman CWN, Habbema JDF, de Jong FH, et al. Serum antimüllerian hormone levels best reflect the reproductive decline with age in normal women with proven fertility: a longitudinal study. Fertility and Sterility 2005;83(4): 979-87.

* Indicates the major publication for the study

\title{
A P P E N D I C E S
}

\section{Appendix I. Cochrane Gynaecology and Fertility specialised register search strategy}

\author{
PROCITE platform
}

From inception to present

Keywords CONTAINS "salpingectomy" or "salpingo-oopherectomy" or Title CONTAINS "salpingectomy" or "salpingo-oopherectomy" 


\section{Appendix 2. Cochrane Central register of controlled trials (CENTRAL) search strategy}

The Cochrane Library platform

From inception to present

1 MeSH descriptor: [Salpingectomy] this term only

2 salpingectom*:ti,ab,kw

3 ((tubal adj2 excision?) or tubectom*):ti,ab,kw

$4 \# 1$ or \#2 or \#3

5 MeSH descriptor: [Ovarian Neoplasms] this term only

6 (ovar* (serou* or cancer* or carcinom* or neoplas* or malignanc* or malignant)):ti,ab,kw

7 MeSH descriptor: [Postoperative Complications] explode all trees

8 Postoperative complication?:ti,ab,kw

$9 \mathrm{MeSH}$ descriptor: [Intraoperative Complications] explode all trees

10 Intraoperative Complication?:ti,ab,kw

11 peroperative complication?:ti,ab,kw

12 surgical injur*:ti,ab,kw

$13 \mathrm{MeSH}$ descriptor: [Blood Loss, Surgical] explode all trees

14 Surgical blood los*:ti,ab,kw

15 surgical hemorrhage?:ti,ab,kw

16 MeSH descriptor: [Infection] explode all trees

17 infection?:ti,ab,kw

18 MeSH descriptor: [Ovarian Reserve] explode all trees

19 ovarian reserve?:ti,ab,kw

20 MeSH descriptor: [Follicle Stimulating Hormone] explode all trees

21 Follicle Stimulating Hormone:ti,ab,kw

22 Follicle-Stimulating Hormone:ti,ab,kw 
23 fsh:ti,ab,kw

24 follitropin:ti,ab,kw

$25 \mathrm{MeSH}$ descriptor: [Primary Ovarian Insufficiency] explode all trees

26 ovarian insufficiency:ti,ab,kw

27 ovarian failure:ti,ab,kw

28 MeSH descriptor: [Anti-Mullerian Hormone] explode all trees

29 (anti mullerian or mullerian inhibiting or mullerian regression or $\mathrm{AMH}$ ):ti,ab, $\mathrm{kw}$

$30 \quad\{$ or \#5-\#29\}

$31 \quad \# 4$ and \#30

\section{Appendix 3. MEDLINE search strategy}

\section{Ovid platform}

From inception to present

1 salpingectom*.ti,ab.

2 salpingectom*.kf.

3 Salpingectomy/

4 Prophylactic Surgical Procedures/ and (fallopian or tubal or tubes).tw.

5 (Prophyla* and (fallopian or tubes or tubal)).tw.

6 (opportunistic and (fallopian or Tubes or tubal)).tw.

7 ((tubal adj2 excision?) or tubectom*).ti,ab.

8 ((tubal adj2 excision?) or tubectom*).kf.

91 or 2 or 3 or 4 or 5 or 6 or 7 or 8

10 Ovarian Neoplasms/

11 (ovar* adj3 (serou* ${ }^{*}$ or cancer* ${ }^{*}$ or carcinom* ${ }^{*}$ or neoplas* or malignanc* or malignant)).ti,ab.

12 (ovar* adj3 (serou* or cancer* or carcinom* or neoplas* or malignanc* or malignant)).kf.

1310 or 11 or 12

14 Postoperative Complications/

15 Postoperative complication?.ti,ab.

16 Postoperative complication?.kf.

17 Intraoperative Complications/

18 Intraoperative Complication?.ti,ab.

19 Intraoperative Complication?.kf.

20 peroperative complication?.ti,ab.

21 peroperative complication?.kf.

22 surgical injur*.ti,ab.

23 surgical injur*.kf.

24 Blood loss, surgical/

25 Surgical blood los*.ti,ab.

26 Surgical blood los*.kf. 
27 surgical hemorrhage?.ti,ab.

28 surgical hemorrhage?.kf.

29 infection/

30 infection?.ti,ab.

31 infection?.kf.

3214 or 15 or 16 or 17 or 18 or 19 or 20 or 21 or 22 or 23 or 24 or 25 or 26 or 27 or 28 or 29 or 30 or 31

33 Ovarian reservel

34 ovarian reserve?.ti,ab.

35 ovarian reserve?.kf.

36 Follicle Stimulating Hormone/

37 Follicle Stimulating Hormone.ti,ab.

38 Follicle Stimulating Hormone.kf.

39 fsh.ti,ab

40 fsh.kf.

41 follitropin.ti,ab.

42 follitropin.kf.

43 primary ovarian insufficiency/

44 ovarian insufficiency.ti,ab.

45 ovarian insufficiency.kf.

46 Anti-mullerian hormone/

47 (anti?mullerian or mullerian?inhibiting or mullerian regression or AMH).ti,ab.

48 (anti?mullerian or mullerian?inhibiting or mullerian regression or $\mathrm{AMH}$ ).kf.

49 ovarian failure.ti,ab.

50 ovarian failure.kf.

5133 or 34 or 35 or 36 or 37 or 38 or 39 or 40 or 41 or 42 or 43 or 44 or 45 or 46 or 47 or 48 or 49 or 50

5213 or 32 or 51

539 and 52

\section{Appendix 4. Embase search strategy}

Ovid platform

From inception to present

1 salpingectom*.ti,ab.

2 Salpingectomy/

3 prophylactic surgical procedure/ and (fallopian or tubal or tubes).tw.

4 (Prophyla* and (fallopian or tubes or tubal)).tw.

5 (opportunistic and (fallopian or Tubes or tubal)).tw.

6 ((tubal adj2 excision?) or tubectom*).ti,ab.

7 (tubes adj2 excision*).ti,ab,kw.

8 or/ $1-7$

9 ovary tumor/

10 (ovar* $^{*}$ adj3 (serou* or cancer* $^{*}$ or carcinom* or neoplas* or malignanc* $^{*}$ or malignant)).ti,ab,kw.

11 (ovar* adj3 tumo? r $^{*}$ ).ti,ab,kw.

12 or $/ 9-11$

13 postoperative complication/

14 Postoperative complication?.ti,ab,kw.

15 peroperative complication/

16 Intraoperative Complication?.ti,ab,kw.

17 peroperative complication?.ti,ab,kw.

18 surgical injur*.ti,ab,kw.

19 operative blood loss/

20 Surgical blood los*.ti,ab,kw.

Hysterectomy with salpingectomy versus hysterectomy alone (Protocol)

Copyright ( 2017 The Cochrane Collaboration. Published by John Wiley \& Sons, Ltd. 
21 surgical hemorrhage?.ti,ab,kw.

22 abdominal infection/ or infection/

23 infection?.ti,ab,kw.

24 or/ $13-23$

25 ovary function/ or ovarian reserve/

26 ovarian reserve?.ti,ab,kw.

27 follitropin/

28 Follicle?Stimulating Hormone.ti,ab,kw.

29 Follicle Stimulating Hormone.ti,ab,kw.

30 fsh.ti,ab,kw.

31 follitropin.ti,ab,kw.

32 ovary insufficiency/ or premature ovarian failure/

33 ovarian insufficiency.ti,ab, kw.

34 ovarian failure.ti,ab,kw.

35 Muellerian inhibiting factor/

36 (anti?mullerian or mullerian?inhibiting or mullerian regression or $\mathrm{AMH}$ ).ti,ab,kw.

37 or/ $25-36$

3812 or 24 or 37

398 and 38

\section{Appendix 5. PsycINFO search strategy}

Ovid platform

From inception to present

1 salpingectom*.tw.

2 ((tubal adj2 excision*) or tubectom* $\left.{ }^{*}\right)$.tw.

31 or 2

\section{Appendix 6. CINAHL search strategy}

Ebsco platform

From inception to present

\section{\# Query}

S9 S5 AND S8

S8 $\quad$ S6 OR S7

S7 TX hysterectom*

S6 (MM “Hysterectomy+")

S5 S1 OR S2 OR S3 OR S4

S4 TX (tubes N2 excision*)

S3 TX ((tubal N2 excision*) or tubectom*) 
S2 TX salpingectom*

S1 (MM "Salpingectomy")

\section{Appendix 7. Clinical trial registries search strategy}

Web platform

From inception to present

'Salpingectomy AND hysterectomy'

\section{CONTRIBUTIONSOFAUTHORS}

JP conceived the idea for this study. The objectives and methods were further clarified by JP, JdH, MV, JW, SH, LvL and MS. JP, LvL and MS drafted the protocol. JP, JdH, MV, JW and SH reviewed the protocol and provided critical feedback.

\section{DECLARATIONSOF INTEREST}

JP, JdH, MV, JW, SH, LvL and MS have no interests to declare.

\section{SOURCES OF SUPPORT}

\section{Internal sources}

- None, Other.

\section{External sources}

- None, Other. 